\section{Commentary: Risk score for death or transplantation after stage I palliation-Now what?}

\author{
Bahaaldin Alsoufi, MD
}

In the current issue of the Journal, Ahmed and colleagues ${ }^{1}$ report a study in which they examined patients enrolled in the National Pediatric Cardiology Quality Improvement Collaborative (NPC-QIC) registry from 2008 to 2015, with a focus on those who had interstage death occur $(153 / 2128 ; 7 \%)$ or received heart transplants $(42 / 2128$; $2 \%$ ). They split the patients into a learning cohort ( $\mathrm{n}=1596)$, from which they derived a risk score for death or transplantation after discharge from the stage I operation, and a validation cohort $(n=532)$, on which they tested and confirmed the strength of their risk score. On the basis of multivariable logistic regression analysis, Ahmed and colleagues ${ }^{1}$ identified the following risk factors, which they used in the risk score: stage I surgery type (hybrid or Norwood procedure with aortopulmonary shunt vs Norwood procedure with right ventricle to pulmonary artery shunt), postoperative extracorporeal membrane oxygenation support, discharge on opiates, no digoxin on discharge, postoperative arch obstruction, and moderate to severe postoperative tricuspid valve regurgitation (with or without oxygen requirement). After Ahmed and colleagues ${ }^{1}$ added weights to those factors according to the related odds ratios, the risk score ranged from 0 to 76 , and a value greater than 17 was associated with a nearly 3 -fold increase in the risk of death or transplantation in both learning and validation cohorts.

Interstage death continues to be a significant problem, as by extension does failure to progress to succeeding palliation stage, necessitating listing for heart transplantation. Consequently, it makes great sense to identify risk factors

\footnotetext{
From the Department of Cardiovascular and Thoracic Surgery, University of Louisville School of Medicine, Norton Children's Hospital, Louisville, Ky.

Disclosures: Author has nothing to disclose with regard to commercial support.

Received for publication Nov 19, 2019; accepted for publication Nov 19, 2019; available ahead of print Dec 9, 2019.

Address for reprints: Bahaaldin Alsoufi, MD, Department of Cardiovascular and Thoracic Surgery, University of Louisville School of Medicine, Norton Children's Hospital, 201 Abraham Flexner Way, Suite 1200, Louisville, KY 40202 (E-mail: balsoufi@hotmail.com).

J Thorac Cardiovasc Surg 2020;160:1031-2

$0022-5223 / \$ 36.00$

Copyright (C) 2019 by The American Association for Thoracic Surgery

https://doi.org/10.1016/j.jtcvs.2019.11.093
}

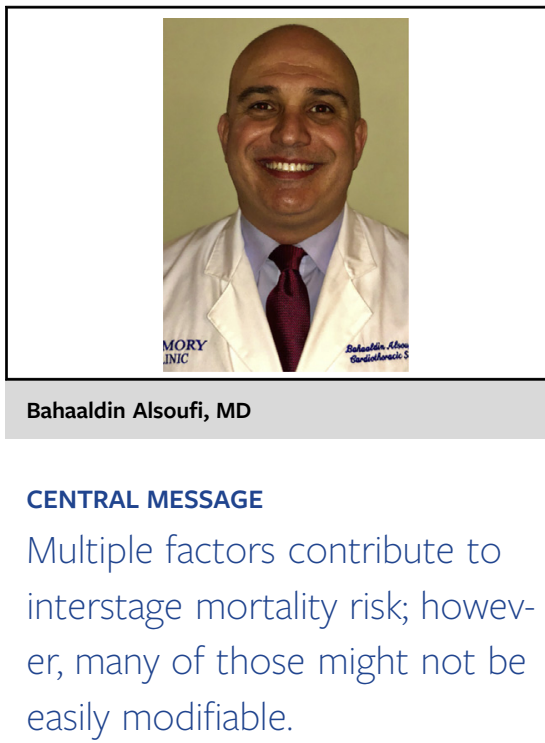

associated with those events, with the aim of neutralizing some of them if possible. The efforts by Ahmed and colleagues ${ }^{1}$ to outline patients at risk are therefore commendable. Early failure of the multistage palliation strategy and its consequent mortality risk, however, are often complex issues related to numerous interlocking variables, making a risk score seem an oversimplification of a complicated matter. If we were to rely on this score exclusively to determine the risk of death or transplantation and to modify our discharge or outpatient follow-up plan accordingly, we might be ignoring several vital factors that did not make the cut in this score because of lack of statistical power or dilution of effect by operative mortality. Examples of those omitted factors include prematurity, low weight, genetic syndromes and extracardiac anomalies, dominant ventricular dysfunction, and history of pulmonary vein intervention or restrictive atrial communication. ${ }^{2-5}$ In addition, there are often incalculable factors that, although likely contributing to death however are naturally immeasurable, thus prohibiting their inclusion in the risk score. Examples of those include family compliance, quality of home monitoring, shunt and pulmonary artery characteristics, aspiration, and arrhythmias. These clinically important issues should be taken into consideration on hospital discharge and during outpatient follow-up, and a thoughtful decision should be made individually for each patient on the basis of numerous potential factors that extend beyond those few entered in the risk score.

On the other hand, the current risk score of Ahmed and colleagues ${ }^{1}$ highlights some of the important risk factors associated with death or transplantation after hospital 
discharge. Whether those factors are modifiable remains a question. Stage I operation type is largely surgeon dependent, and given that the Single Ventricle Reconstruction study showed no advantageous effect of shunt type on overall survival at 3 or 6 years after the Norwood procedure, it is unlikely that surgeons will change their approach, especially given that the odds ratio and subsequently the weight that this surgery type holds in the proposed risk score are low (weight of 3). ${ }^{4,5}$ Moreover, patients who underwent the hybrid approach in the registry were few (only $7 \%$ ), and they likely had associated risk factors, thus complicating the interpretation of the results in the current study of Ahmed and colleagues ${ }^{1}$ and making it unlikely that surgeons who use the hybrid strategy selectively in neonates at higher risk will change their strategy in complex cases. Residual arch obstruction is a straightforward issue and generally amenable to percutaneous or surgical intervention, and the decision to repair this residual lesion is therefore clear and direct. On the other hand, the remaining factors in this score might not be as easily modifiable. Discharge on opiates is likely a surrogate for a prolonged and complex postoperative course, and such patients could have lingering cardiac and extracardiac issues that might increase their risk after discharge. Similarly, postoperative extracorporeal membrane oxygenation use after the stage I operation is a well-documented factor for early and interstage mortality, and the postdischarge attrition risk is generally attributed to residual lesions, continuous dysfunction, and end-organ injury. ${ }^{6,7}$ Although correction of residual lesions is an important principle to improve outcomes in these patients, the remaining issues related to myocardial and end-organ dysfunction are hardly amendable. Finally, the issue of tricuspid valve regurgitation is complex and difficult to address. Although there is general consensus that moderate to severe tricuspid valve regurgitation after the stage I operation is problematic, management options at this stage are limited, making the likelihood of effectively modifying this factor more theoretic than practical. Tricuspid valve repair during the neonatal period is challenging because of the frequent presence of structural valve problems when significant regurgitation is present at that early stage, as well as tissue friability that often precludes successful repair. Multiple reports have demonstrated that tricuspid valve repair before the stage II operation is associated with high mortality and failure risk and frequent deterioration in systemic right ventricular function. ${ }^{8-10}$ Tricuspid valve repair is therefore commonly reserved for those with failed recovery after the stage I operation, and it would be unlikely for surgeons to attempt tricuspid valve surgery in neonates with moderate to severe tricuspid valve regurgitation who are thriving and otherwise candidates for hospital discharge. Continuous hospitalization until the stage II operation and early listing of proper candidates for heart transplantation might be a consideration in those cases. Although the practice of early listing for transplantation might decrease interstage death, it is also likely to simultaneously increase the number of patients receiving transplantation, the other arm in the composite end point that Ahmed and colleagues $^{1}$ examined.

In summary, the risk score proposed by Ahmed and colleagues ${ }^{1}$ covers some but not all of the clinically important risk factors that affect interstage mortality and transplant risk after the stage I operation. Although awareness of those risk factors is important, unfortunately, many of those factors are not easily modifiable.

\section{References}

1. Ahmed H, Anderson JB, Bates KE, Fleishman CE, Natarajan S, Ghanayem NS, et al. Development of a validated risk score for interstage death or transplant after stage I palliation for single ventricle heart disease. J Thorac Cardiovasc Surg. 2020;160:1021-30.

2. Alsoufi B, McCracken C, Kochilas LK, Clabby M, Kanter K. Factors associated with interstage mortality following neonatal single ventricle palliation. World $J$ Pediatr Congenit Heart Surg. 2018;9:616-23.

3. Alsoufi B, Mori M, Gillespie S, Schlosser B, Slesnick T, Kogon B, et al. Impact of patient characteristics and anatomy on results of Norwood operation for hypoplastic left heart syndrome. Ann Thorac Surg. 2015;100:591-8.

4. Newburger JW, Sleeper LA, Gaynor JW, Hollenbeck-Pringle D, Frommelt PC, Li JS, et al; Pediatric Heart Network Investigators. Transplant-free survival and interventions at 6 years in the SVR Trial. Circulation. 2018;137:2246-53.

5. Ghanayem NS, Allen KR, Tabbutt S, Atz AM, Clabby ML, Cooper DS, et al; Pediatric Heart Network Investigators. Interstage mortality after the Norwood procedure: results of the multicenter Single Ventricle Reconstruction Trial. J Thorac Cardiovasc Surg. 2012;144:896-906.

6. Alsoufi B, Wolf M, Botha P, Kogon B, McCracken C, Ehrlich A, et al. Late outcomes of infants supported by extracorporeal membrane oxygenation following the Norwood operation. World J Pediatr Congenit Heart Surg. 2015; 6:9-17.

7. Friedland-Little JM, Aiyagari R, Yu S, Donohue JE, Hirsch-Romano JC. Survival through staged palliation: fate of infants supported by extracorporeal membrane oxygenation after the Norwood operation. Ann Thorac Surg. 2014;97:659-65.

8. Honjo O, Atlin CR, Mertens L, Al-Radi OO, Redington AN, Caldarone CA, et al. Atrioventricular valve repair in patients with functional single-ventricle physiology: impact of ventricular and valve function and morphology on survival and reintervention. J Thorac Cardiovasc Surg. 2011;142:326-35.e2.

9. Alsoufi B, Sinha R, McCracken C, Figueroa J, Altin F, Kanter K. Outcomes and risk factors associated with tricuspid valve repair in children with hypoplastic left heart syndrome. Eur J Cardiothorac Surg. 2018;54:993-1000.

10. Nakata T, Fujimoto Y, Hirose K, Tosaka Y, Ide Y, Tachi M, et al. Atrioventricular valve repair in patients with functional single ventricle. J Thorac Cardiovasc Surg. 2010;140:514-21. 